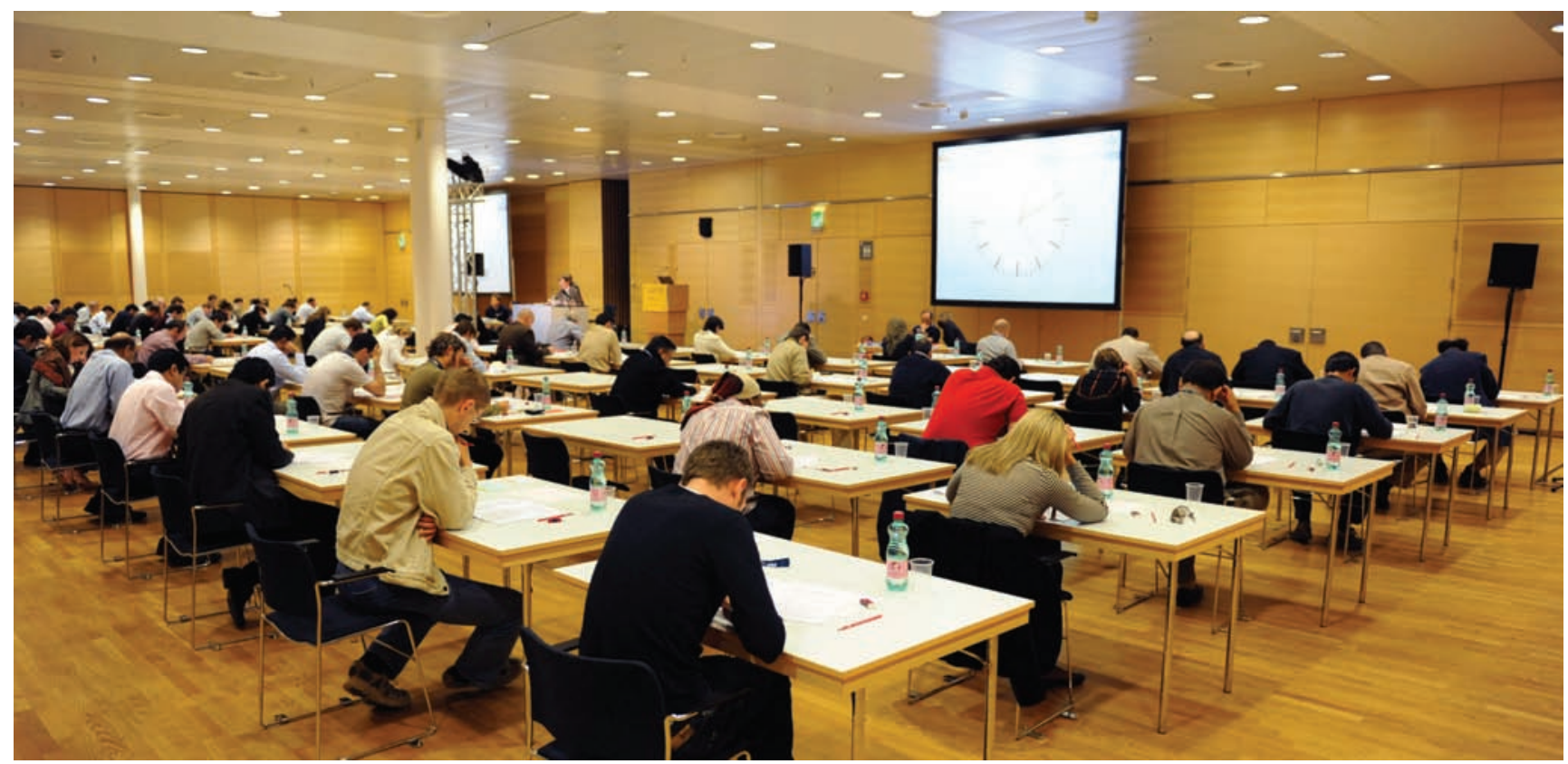

\title{
HERMES examinations in adult and paediatric respiratory medicine
}

The HERMES examinations in adult and paediatric respiratory medicine mark significant milestones in the overall mission to Harmonise Education in Respiratory Medicine for European Specialists. The knowledge-based examinations originate from the corresponding HERMES syllabi in adult respiratory medicine and paediatric respiratory medicine, covering 90 multiple choice questions taken over 3 hours at the ERS Annual Congress. Individuals now have the opportunity for a number of options should they wish to sit the examination

In-training assessment: for those currently within their specialist training period

Self assessment: open to all medical practitioners who would like to benchmark themselves

European Diploma: for those who are nationally accredited adult or paediatric respiratory specialists

Detailed individual feedback according to syllabus topics will be provided to allow participants to assess progress and as a guide to assign priorities for further studies and continuous education. The 2011 HERMES examinations will take place in Amsterdam on September 24th at 14:00. More information can be found at http://hermes.ersnet.org/

\section{HERMES examination in adult respiratory medicine}

Approaching the fourth year of the HERMES exam in adult respiratory medicine, the exam continues to gain merit, esteem and recognition. The HERMES exam is recognised officially by the Swiss as the written part of their official exit examination in adult respiratory medicine for their trainees. The Netherlands too has endorsed recognition and Austria is the most recent country to formally recognise the HERMES examination as equivalent to their official national exam. Further campaigning

\section{S. Mitchell}

ERS, Lausanne, Switzerland

\section{Correspondence}

S. Mitchell

Avenue Sainte-Luce 4

1003 Lausanne

Switzerland

Sharon.mitchell@ersnet.org

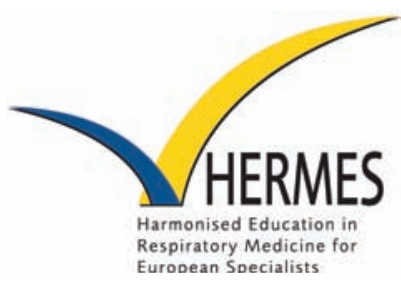


has lead to discussions of collaboration with additional countries both in and outside of Europe.

As the number of candidates who are awarded the European diploma increases each year, so too does the acclaim of HERMES. Generally, those who have sat the examination have been in agreement with Dr. Ali Elsheikh, who stated that taking the HERMES examination "proved to be a very positive experience". The mentorship scheme also allows those who have successfully passed the examination to offer advice to candidates hoping to pass and become a HERMES diplomate.

In support of the HERMES initiative, a number of educational resources continue to be developed. The ERS Handbook of Respiratory Medicine is the most recent edition, presenting an overview of each of the modules listed in the syllabus. Preparations for the development of an examination guide including multiple choice questions and answer explanations are now being made, further strengthening support for those preparing to take the exam.

\section{HERMES examination in paediatric respiratory medicine}

The inaugural examination in paediatric respiratory medicine will take place in Amsterdam on September 24th. It is hoped that the paediatric examination will follow the successful footsteps of the HERMES examination in adult respiratory medicine, and that the benefits of becoming a HERMES diplomate are realised.

\section{Proof of excellence}

As an experienced physician, or even if you are still at the beginning of your career, the ERS European Diploma in Respiratory Medicine will allow you to show both your patients and colleagues your commitment to high-quality lifelong learning.

\section{Mobility}

If you are at a stage of your career when you think of moving to another country, this European Diploma, even if not legally recognised by most national health authorities, will represent a mark of excellence throughout the profession, thus making moving and working in other countries easier.

\section{Self assessment}

No matter how experienced you are, it might be interesting for you to sit this examination in order to find out where you stand with respect to what is regarded as the current European gold standard.

\section{Tangible recognition}

Upon successful completion of the examination, the ERS will award you a European Diploma in Respiratory Medicine. You will also belong to the HERMES Diplomates Registry reserved for successful candidates, which will provide enhanced networking possibilities and other benefits. Diplomates will also have the opportunity to update their qualification at intervals throughout their career to confirm their continuing high quality. 